\title{
An Ultra-Compact Design of Plasmonic Memristor with Low Loss and High Extinction Efficiency Based on Enhanced Interaction between Filament and Concentrated Plasmon
}

\author{
Ye Tian ${ }^{1,2,3, *}$, Saiwen Zhang ${ }^{1}$ and Weishi Tan ${ }^{1}$ \\ 1 School of Information and Electronic Engineering, Hunan City University, Yiyang 413000, China; \\ zhangsaiwen@hncu.edu.cn (S.Z.); tanweishi@mail.njust.edu.cn (W.T.) \\ 2 Chongqing United Microelectronics Center, Chongqing 410332, China \\ 3 All-Solid-State Energy Storage Materials and Devices Key Laboratory of Hunan Province, \\ Yiyang 413000, China \\ * Correspondence: tianye-man@163.com
}

Citation: Tian, Y.; Zhang, S.; Tan, W. An Ultra-Compact Design of Plasmonic Memristor with Low Loss and High Extinction Efficiency Based on Enhanced Interaction between Filament and Concentrated Plasmon. Photonics 2021, 8, 437. https:// doi.org/10.3390/photonics 8100437

Received: 4 September 2021

Accepted: 7 October 2021

Published: 12 October 2021

Publisher's Note: MDPI stays neutral with regard to jurisdictional claims in published maps and institutional affiliations.

Copyright: (c) 2021 by the authors. Licensee MDPI, Basel, Switzerland. This article is an open access article distributed under the terms and conditions of the Creative Commons Attribution (CC BY) license (https:// creativecommons.org/licenses/by/ $4.0 /)$.

\begin{abstract}
We present a numerical design of the plasmonic memristive switching device operated at the telecommunication wavelength of $1.55 \mu \mathrm{m}$, which consists of a triangle-shaped metal taper mounted on top of a Si waveguide, with rational doping in the area below the apex of the taper. This device can achieve optimal vertical coupling of light energy from the Si waveguide to the plasmonic region and, at the same time, focus the plasmon into the apex of the metal taper. Moreover, the area with concentrated plasmon is overlapped with that where the memristive switching occurs, due to the formation/removal of the metallic nano-filament. As a result, the highly distinct transmission induced by the switching of the plasmonic memristor can be produced because of the maximized interactions between the filament and the plasmon. Our numerical simulation shows that the device hasa compact size $(610 \mathrm{~nm})$, low insertion loss $(\sim 1 \mathrm{~dB})$, and high extinction efficiency $(4.6 \mathrm{~dB} / \mu \mathrm{m})$. Additionally, we point out that stabilizing the size of the filament is critical to improve the operation repeatability of the plasmonic memristive switching device.
\end{abstract}

Keywords: silicon photonics; plasmonics; neuromorphic computing; nano-filament

\section{Introduction}

Integrated photonic chips have been widely studied and applied in photonic science and engineering, which can efficiently speed up on-chip information processing [1]. However, owing to the diffraction limit, it is difficult to realize integrated photonic circuits with a density comparable to the electronic counterpart [1]. In recent years, much research attention has been paid to plasmonic devices, which have the capability of controlling the light fieldon a deep subwavelength scale [2-6]. Along with the good development of the components for the propagation, modulationand detection of plasmon [7-9], explorations of plasmonic devices for logic operation and data storage are also on the road, e.g., recently, surface plasmon polaritons (SPP) were introduced into the memristor, making its resistive state optical readable [10]. This is from the perspective that the combination of plasmon and the memristor might induce unique opportunities for new functional plasmonic devices.

The memristor is a device that can be tuned between a high-resistance (HR) state and low-resistance (LR) state by the external voltage/current signal (Figure 1a) [11]. It typically consists of a capacitor-like metal-insulator-metal (MIM) or metal-insulator-semiconductor (MIS) structure [11-13], which naturallycould also serve as the plasmonic waveguide [14]. Hence, interestingly, the memristor in an HR or LR state could exhibit a discrepant optical response when interacting with the plasmon, as shown in Figure $1 b[10,14,15]$. This system (namely, "plasmonic memristor"), on one hand, has volatile or non-volatile memory with electrical write/erase and optical readout functionality $[10,15,16]$. On the other 
hand, noting the potential of the memristor as a basic unit for a chip with the capability of neuron computation and implication logical operation $[17,18]$, many of the applications, such as in-memory optical computing, optoelectronic artificial synapses, image recognition and even neuromorphic visual systems, were explored at different wavelength ranges $[10,14,15,19-23]$. The plasmonic memristor based on a silicon photonics platform is especially attractive, due to its emerging chances to realize large-scale interrogation via a CMOS compatible process $[10,14,15,19,20]$, as well as its potential to fuse its data processing capability with the strong power of silicon photonics on data transmission at communication wavelengths around $1.31 \mu \mathrm{m}$ and $1.55 \mu \mathrm{m}[19,20]$.
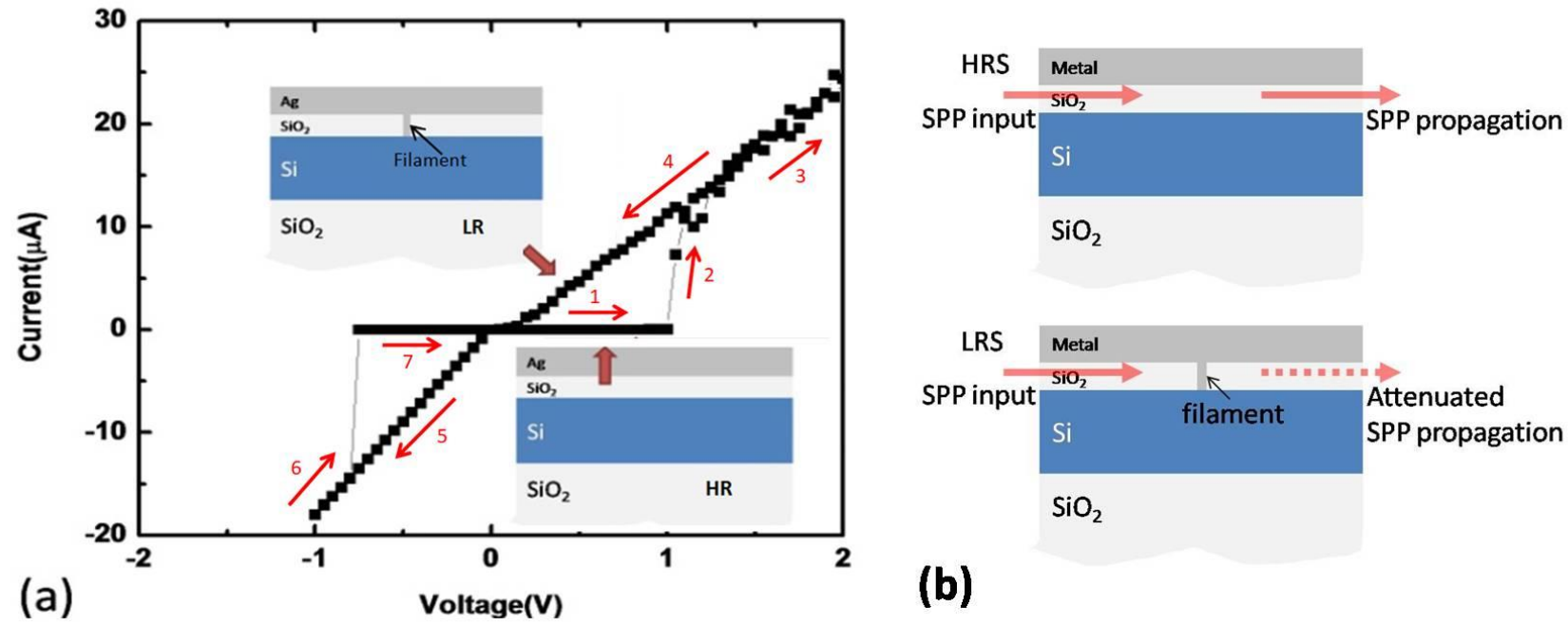

(b)
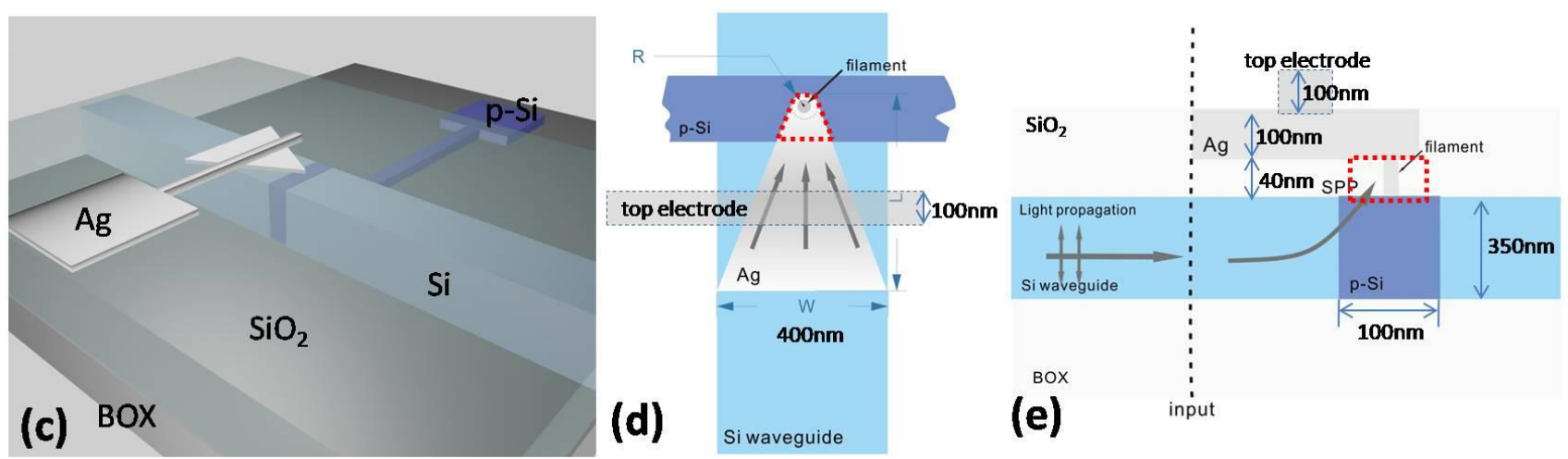

Figure 1. (a) Memristive behaviors of $\mathrm{Ag} / \mathrm{SiO}_{2} / \mathrm{Si}$ trilayer system; (b) conceptual illustration of the distinct interaction between plasmon and memristor at HR (upper) and LR (bottom) state; (c) three-dimensional visualization of the designed plasmonic memristor based on triangle-shaped metal taper on top of a dielectric wave guide (BOX: buried oxide); (d) top view; and (e) side view of the plasmonic memristor.

There are many memristive mechanisms [24-26], which enable the use of optical memristive switches at the micro- to atomic scale [16]. In the plasmonic memristor based on the formation/rupture of the metallic filament in the insulator, its filament induces absorption and scattering in small volumes at the nano- or even atomic scale [10,14].This, in principle, is quite beneficial to shrink the size of the plasmonic memristor. In this work, we numerically design a plasmonic memristor operating at a telecom wavelength of $1.55 \mu \mathrm{m}$, based on filament formation/rupture. The simulation shows that the proposed structure is rather compact (width $\sim 400 \mathrm{~nm}$, length $\sim 610 \mathrm{~nm}$ ), and with a relative low loss and high extinction efficiency $[10,14]$. 


\section{Experimental and Methods}

The basic structure of the device proposed in this work is a small triangle-shaped $\mathrm{Ag}$ taper on top of a silicon ( $\mathrm{Si}$ ) waveguide, as shown in Figure 1c. The substrate is silicon dioxide $\left(\mathrm{SiO}_{2}\right)$, and a $\mathrm{SiO}_{2}$ buffer layer is used to separate the $\mathrm{Ag}$ triangle and the $\mathrm{Si}$ waveguide. Such an MIS $\left(\mathrm{Ag} / \mathrm{SiO}_{2} / \mathrm{Si}\right)$ structure could guide the plasmon, but to ensure the device could truly work similarly to a plasmonic memristor, we still experimentally pre-check the memristive behaviors of the $\mathrm{Ag} / \mathrm{SiO}_{2} / \mathrm{Si}$ tri-layered system. Its preparation starts from the p-doped $\mathrm{Si}$ substrate, which is ultrasonically cleaned successively in acetone, ethanol, acetone, ethanol, and deionized water for $10 \mathrm{~min}$ each, then blown dry by $\mathrm{N}_{2}$ flow and baked in vacuum oven at $120{ }^{\circ} \mathrm{C}$ for $2 \mathrm{~h}$. A layer ofSiO $\mathrm{O}_{2}$ with thickness of $\sim 30 \mathrm{~nm}$ is deposited by e-beam evaporation (BOC Edward FL400) on the cleaned silicon chip, which is dipped in $10 \% \mathrm{HF}$ solution and blown dry by $\mathrm{N}_{2}$ flow to remove the natural surface oxide layer. After that, the top electrode Ag layer with $100 \mathrm{~nm}$ thickness is defined by lithography plus lift-off process (SUSS MJB4). The electrical tests on the obtained sample are performed on Keithley-2635A sourcemeter. A voltage sequence $(0 \mathrm{~V} \rightarrow 2 \mathrm{~V} \rightarrow-1 \mathrm{~V} \rightarrow 0 \mathrm{~V})$ is applied on the $\mathrm{Ag} / \mathrm{SiO}_{2} / \mathrm{Si}$ trilayer structure and the current is probed, where the positive voltage is applied from $\mathrm{Ag}$ to $\mathrm{Si}$ (serves as the grounded bottom electrode). On the other hand, the numerical design of the plasmonic memristor containing $\mathrm{Ag} / \mathrm{SiO}_{2} / \mathrm{Si}$ trilayer structure is performed in FDTD solution and mode solution from Ansys-Lumerical.

\section{Results and Discussions}

\subsection{The Device Working Principle}

As shown in Figure 1a, as the positive voltage reaches $\sim 1 \mathrm{~V}$, the current through the device presents a sudden increase, which corresponds to the switching of the $\mathrm{Ag} / \mathrm{SiO}_{2} / \mathrm{Si}$ system from an HR to LR state; while, in the negative voltage regime, a voltage $\sim-0.8 \mathrm{~V}$ could produce the reverse switching from the LR to HR state. Such inter-switching between the HR and LR state, under a rational electrical field, is a typical memristive phenomenon, verifying the $\mathrm{Ag} / \mathrm{SiO}_{2} / \mathrm{Si}$ trilayer structure could be a good memristor. Moreover, the IV characteristic of the $\mathrm{Ag} / \mathrm{SiO}_{2} / \mathrm{Si}$ device in the LR state seems highly ohmic, which is a conductive feature of the metal and strongly implies the formation of a metal filament during the switching. Hence, without the loss of rationality, we assume that the $\mathrm{Ag} / \mathrm{SiO}_{2} / \mathrm{Si}$ memristor is based on a metal filament formation/rupture mechanism. Under this precondition, the $\mathrm{Ag} / \mathrm{SiO}_{2} / \mathrm{Si}$ system with a triangle-shaped $\mathrm{Ag}$ taperis employed to design the plasmonic memristor because it is a memristor and could simultaneously support the HP mode in the $\mathrm{SiO}_{2}$ buffer layer.

In this system, it has been observed that the plasmon can be concentrated to the area below the apex of the taper (Figure 1d, the top view of the structure), while the loss is low and the size is small $[27,28]$. Hence, if the length of the taper is also chosen to maximize the energy transfer from the incident $\mathrm{Si}$ waveguide to plasmonic taper (Figure 1e, the side view of the structure) [28], these two factors together ensure that the electromagnetic energy of the incident light could be tightly focused on the $\mathrm{SiO}_{2}$ buffer layer at the area below the apex of the taper, with a small volume. Furthermore, the Ag taper can also be employed as the top electrode of the plasmonic memristor, and connected via the Ag line on its upper side, while the bottom electrode is the silicon with p-doping in the area below the apex of the taper (note that the $\mathrm{Ag}$ taper, the $\mathrm{SiO}_{2}$ buffer layer, and the $\mathrm{Si}$ waveguide together form the memristive $\mathrm{Ag} / \mathrm{SiO}_{2} / \mathrm{Si}$ trilayer structure; the heavy doping level to the silicon at the $\sim 10^{20} \mathrm{~cm}^{-3}$ level is suggested to minimize series resistance [29]). Since the memristive behavior (the filament forms or dissolves) would just take place in the area sandwiched by the top and bottom electrode $[24,26]$, i.e., the zone in the $\mathrm{SiO}_{2}$ buffer layer below the apex of the taper (as marked by a red dotted line in Figure 1d,e), the memristive area would be exactly overlapped with the concentrated light. As a result, the interactions between the plasmon and the filament are maximized to obtain a high extinction efficiency. Meanwhile, the device has a compact size (as discussed below), and, consequently, low loss. This is the core thought of our work. 
The geometric structure of the device is shown in its top view (Figure 1d) and side view (Figure 1e). We use $\mathrm{W}$ to denote the length of the base of the isosceles triangle (metal taper), and $\mathrm{L}$ to denote the perpendicular length from the input side to the apex. For simplicity, we will call $\mathrm{W}$ the (maximum) width of the triangle and $\mathrm{L}$ the length of the triangle throughout this paper. Since the apex of the metal triangle is always rounded up in real manufacturing, we introduce the radius of curvature $\mathrm{R}(=20 \mathrm{~nm})$ at the tip, which is a typical feature size feasible by, e.g., a regular e-beam lithography tool, if no special measures are conducted to further enhance the fabrication resolution [30].

\subsection{Mode Analysis of the Plasmonic Memristor}

There are two types of modes existing in the plasmonic taper, TE-like modes and TMlike modes [28], but the TM-like modes are of more of interest because they can be focused at the tip of the triangle, as opposed to the TE modes. Hence, in the following, we naturally focus on the TM-like modes in the taper (Figure 2a). As specified for the incident fundamental TM photonic mode of the Si waveguide at a wavelength of $1550 \mathrm{~nm}$ (Figure 2b,c), the $\mathrm{H}_{\mathrm{TM}, 0}$ and $\mathrm{H}_{\mathrm{TM}, 1}$ super modes can be excited at the input of the plasmonic taper (i.e., at $\mathrm{w}=400 \mathrm{~nm}$, see Figure $2 \mathrm{~d}-\mathrm{g}$ ). The width of the taper gradually decreases from $\mathrm{w}=400 \mathrm{~nm}$ at the input to $\mathrm{w}=0 \mathrm{~nm}$ at the apex (their mode profiles at the place near the apex with a narrow taper width of $40 \mathrm{~nm}$ are shown in Figure $2 \mathrm{~h}-\mathrm{k}$ ), which can be confirmed from the dependence of the effective index of these modes on the taper width, as shown in Figure 21. It can be clearly observed that for either of the $\mathrm{H}_{\mathrm{TM}, 0}$ and $\mathrm{H}_{\mathrm{TM}, 1}$ modes, the electrical field is tightly confined in the $\mathrm{SiO}_{2}$ layer. Meanwhile, their distribution is always under the metal zone, such that the $\mathrm{H}_{\mathrm{TM}, 0}$ and $\mathrm{H}_{\mathrm{TM}, 1}$ modes are highly overlapped with each other in the taper, and, accordingly, they propagate through the plasmonic nano-taper and could couple via directional coupling mechanism [28].

(a)
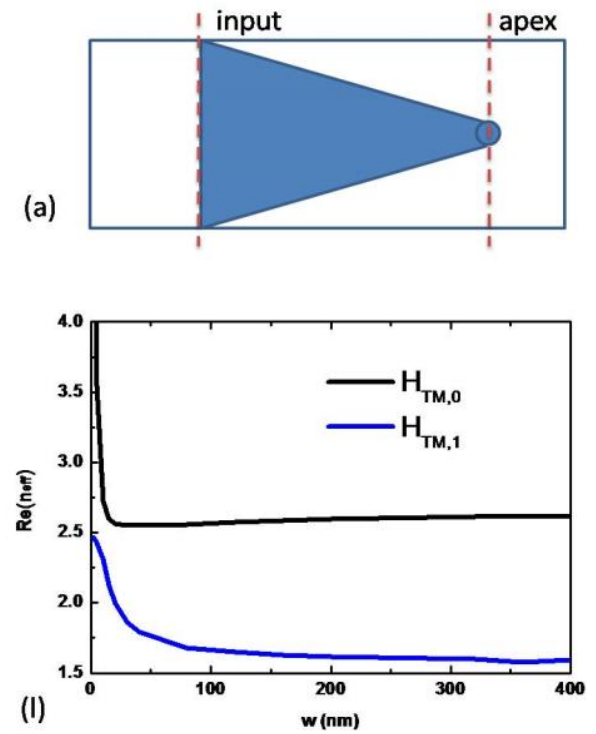

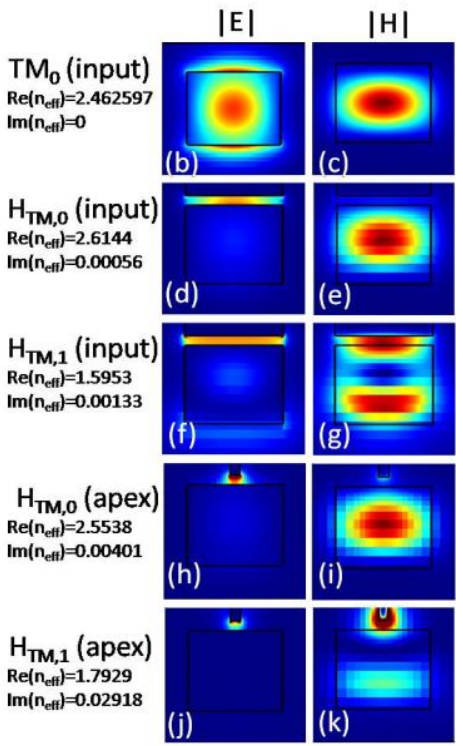

Figure 2. (a) The concerned sites (input and apex as marked by red dashed lines) in the mode analysis to the plasmonic memristor; (b) electrical field and (c) magnetic field profile of the TM mode inputted from Si waveguide; (d) electrical field and (e) magnetic field profile of the $\mathrm{H}_{\mathrm{TM}, 0}$ mode exited at the input of the taper $(\mathrm{w}=400 \mathrm{~nm})$; (f) electrical field and $(\mathrm{g})$ magnetic field profile of the $\mathrm{H}_{\mathrm{TM}, 1}$ mode exited at the input of the taper $(\mathrm{w}=400 \mathrm{~nm}) ;(\mathrm{h})$ electrical field and (i) magnetic field profile of the $\mathrm{H}_{\mathrm{TM}, 0}$ mode propagated to the apex of the taper $(\mathrm{w}=40 \mathrm{~nm}) ;(\mathbf{j})$ electrical field and $(\mathbf{k})$ magnetic field profile of the HTM, 1 mode propagated to the apex of the taper $(\mathrm{w}=40 \mathrm{~nm})$; $(\mathbf{l})$ mode effective indices of $\mathrm{H}_{\mathrm{TM}, 0}$ and $\mathrm{H}_{\mathrm{TM}, 1}$ modes as the function of the width at different sites of silver taper.

As specified for the $\mathrm{H}_{\mathrm{TM}, 0}$ and $\mathrm{H}_{\mathrm{TM}, 1}$ modes, the beat length (BL), given by the length over which the relative phase of the super modes differs by $2 \pi$, is inversely proportional 
to the real part of the difference between their effective indices, and can be obtained as follows [28]:

$$
B L(w)=\lambda / \operatorname{Re}\left(\Delta n_{e f f}(w)\right)
$$

where $\lambda$ is the operation wavelength, and $\Delta n_{\text {eff }}$ is the difference between the effective index of $\mathrm{H}_{\mathrm{TM}, 0}$ and that of $\mathrm{H}_{\mathrm{TM}, 1}$. Obviously, BL decreases as the light propagates along the triangle because the effective index of $\mathrm{H}_{\mathrm{TM}, 0}$ increases steeply as the width of the triangle nanotaper decreases, as shown in Figure 21. Finally, the effective index of $\mathrm{H}_{\mathrm{TM}, 0}$ increases asymptotically to infinity, as the effective index of $\mathrm{H}_{\mathrm{TM}, 1}$ is less than 2.5 (Figure 2l), and, thus, the BL approaches zero at the apex of the triangle taper, where the group velocity asymptotically approaches zero. Consequently, the electromagnetic energy is horizontally focused at the apex of the taper. Such a light localization process is common to most geometry-induced nano-focusing based on structures such as the plasmonic taper shown here [31], and serves as one of the main mechanisms to produce extreme light concentration (Figure 1c). Moreover, we check the light-leaking during the focusing by the adiabatic theory. Especially, using the Wentzel-Kramers-Brillouin (WKB) approximation, the aneikonal parameter (also called adiabatic parameter) can be defined as [31]:

$$
\delta=\left|(d w / d x)\left(d\left(k_{0} n_{e f f}\right)^{-1} / d x\right)\right|
$$

where $k_{0}$ and $n_{\text {eff }}$ are the wavevector in vacuum and the effective index of the $\mathrm{H}_{\mathrm{TM}, 0}$ mode, respectively. The eikonal parameter for the $\mathrm{H}_{\mathrm{TM}, 0}$ mode (the main adiabatically changing mode when the width $w$ is tapered down) is less than $0.5(d w / d x)$ when $\mathrm{w}>16 \mathrm{~nm}$, indicating quite-low light-leaking generated in this geometry focusing process. Besides geometry focusing, another critical factor for the light concentration in our plasmonic memristor is the optimal energy transfer from the dielectric waveguide to the plasmonic taper [28]. In particular, for a taper with length $L$, the following applies:

$$
\int_{0}^{L} \frac{d z}{B L(w)}=\left(\int_{0}^{L} \Delta n_{e f f}(w) d z\right) / \lambda=1 / 2
$$

where $z$ is the position away from the input end, and $w$ is the width of the metal taper at position $z$. Here $w$ is determined by the width at the input end $(W=400 \mathrm{~nm})$ and the taper length $L$ with $w(z)=\mathrm{W}(L-z) / L$, if the shape imperfection due to apex-rounding are ignored. Then, the light energy could be vertically coupled efficiently into the $\mathrm{SiO}_{2}$ buffer layer at the apex of the taper (Figure 1d). The combination of geometry-induced horizontal focusing and optimal energy transfer to the plasmonic taper would maximize the light concentration in the $\mathrm{SiO}_{2}$ buffer layer at the apex of the taper, which is overlapped with the memristive area where the filament could form or dissolve. This is the key to achieving a high extinction efficiency.

\subsection{The Properties of the Plasmonic Memristor with Specified Size}

The performance of the plasmonic memristor can be evaluated by checking its optical transmission at the HR stateand LRstate, as well as comparing their difference via a finitedifference time-domain (FDTD, Lumerical solution) simulation. A mode source $\left(\mathrm{TM}_{0}\right.$, see Figure $2 b$ ) supported by the Si waveguide is feed in at the input side and propagates to the taper apex. When the plasmonic memristor is at the HR state, the incident light will go forwards continuously along the Si waveguide. Consequently, the monitor that detects the output light would receive a strong signal, i.e., the plasmonic memristor at the HR state is highly transparent for the $\mathrm{TM}_{0}$ incident source; while, for the device at the LR state, metal filaments would form in the $\mathrm{SiO}_{2}$ buffer layer, resulting in the light absorbing and scattering. Accordingly, low light transmission is produced. The transmission difference between the HR and LR state could be a qualitative reflection of the plasmon-filament interaction, and could be used to evaluate the performance of the plasmonic memristor. 
Although, in the real device, the size, shape and/or site of the filament could be highly random, without the loss of generality, here, we set our plasmonic memristor at the LR state, with a circular filament in the $\mathrm{SiO}_{2}$ buffer layer under the taper apex, located at the center of the circle, as marked in Figure 2a. The diameter of the filament is set as $\sim 10 \mathrm{~nm}$, according to Refs. [32-34] (the geometric influence of the filament will be discussed in detail in Section 3.5).

The specific size of the taper, as well as the Si waveguide, is as shown in Figure 1c,d; however, since $L$ is governed by an integral equation (Equation (3)), and $n_{\text {eff }}(w)$ in this equation is not given in an analytical form, but from the numerical calculated effective index of the $\mathrm{H}_{\mathrm{TM}, 0}$ and $\mathrm{H}_{\mathrm{TM}, 1}$ modes at different widths $\mathrm{w}$, it is quite difficult to explicitly determine the taper length by Equation (3). However, considering that the BL of the triangular taper can be approached by BL $\left(w_{\text {avg }}\right)$ [28], where $w_{\text {avg }}$ is the average metallic width over the taper length, corresponding to about $1 / 2$ of the maximum width $W$ of the taper (i.e., $w_{\text {avg }} \approx \mathrm{W} / 2=200 \mathrm{~nm}$ ), we obtain the following:

$$
\Delta n_{e f f}\left(w_{\text {avg }}\right) L \approx \lambda / 2 \text { or } L \approx \frac{\lambda}{2 \Delta n_{e f f}\left(w_{\text {avg }}\right)}
$$

It is noteworthy that from the calculation based on Equation (4), we cannot obtain a precise value of the optimal $L$ because $n_{\text {eff }}(w)$ would become very large as $\mathrm{w}$ is small, and the initial phase difference between $\mathrm{H}_{\mathrm{TM}, 0}$ and $\mathrm{H}_{\mathrm{TM}, 1}$ at the input side is not taken into account. As a result, $\int_{0}^{L} \Delta n_{e f f}(w) d z$ would be underestimated by $n_{e f f}\left(w_{\text {avg }}\right) L$, resulting in the overestimation of the rational taper length from Equation (4). However, such an approach, as well as the proportional relationship between the taper length and the wavelength revealed from it, still gives us the clues to obtain the rational initial design and/or the guideline for further structure optimization. The optimized length $L$ is $610 \mathrm{~nm}$ to produce the maximum extinction ratio at the telecommunication wavelength of $1.55 \mu \mathrm{m}$ (the details of the optimization will be discussed, together with the taper length dependence of the properties of the plasmonic memristor, in Section 3.4). In this structure, geometryinduced horizontal focusing can be clearly observed in Figure 3a, and the optimal energy transfer to the plasmonic taper is also achieved (Figure 3b). These two effects co-produce the strong plasmon-filament interaction as the device is switched to the LR state, as shown in Figure 3c,d. From the optical transmission features (Figure 3e), it is found that its LR and HR states correspond to two distinct spectra, $\mathrm{T}_{\mathrm{LR}}(\lambda)$ and $\mathrm{T}_{\mathrm{HR}}(\lambda)$, especially around the C-band $(\sim 1.55 \mu \mathrm{m})$. Accordingly, it produces an extinction ratio (defined as $\left.-101 \mathrm{~g}\left(\mathrm{~T}_{\mathrm{LR}} / \mathrm{T}_{\mathrm{HR}}\right)\right)$ of $2.8 \mathrm{~dB}$ at the wavelength $1550 \mathrm{~nm}$ (Figure $3 \mathrm{f}$ ), equivalent to the extinction efficiency of $4.6 \mathrm{~dB} / \mu \mathrm{m}$. Moreover, as this design has a compact size (overall length is $610 \mathrm{~nm}$ ) much shorter than other structures [10,14,35], the intrinsic propagation loss of the plasmonic taper, positively correlated to its length, is reduced [36], resulting in a quite low loss of typically $\sim 1 \mathrm{~dB}$ around $1550 \mathrm{~nm}$ (Figure 3f). Although the properties of the device discussed in this work are evaluated based on the simulations, and are perhaps not suitable to directly compare with the experimental reports summarized in Table $1[10,14,19,20,35,37]$, the achieved results still imply that introducing the small triangle-shaped metal taper on top of the dielectric waveguide could be a promising design for a plasmonic memristor with superior performances. However, it is noteworthy that at the other important communication window $(1.31 \mu \mathrm{m}$, O-band), the spectra change induced by the memristive switching is much weaker, and in an even shorter wavelength range, and the transmission spectra of the device at the HR state become very close to that at the LR state (see Figures $3 e$ and 4); hence, in the following, we mainly focus on the $1.55 \mu \mathrm{m}$ range. 

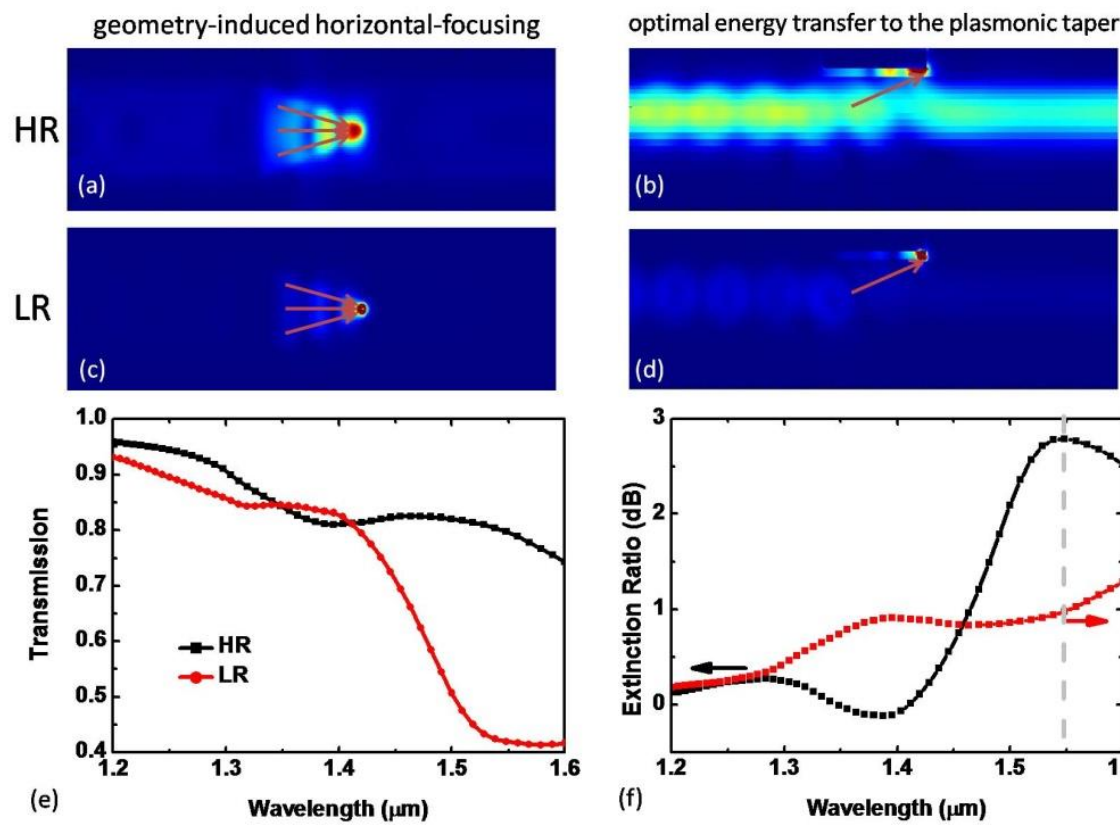

Figure 3. (a) Geometry-induced horizontal focusing and (b) optimal energy transfer to the taper of the plasmonic memristor at HR state; enhanced plasmon-filament interaction in the plasmonic memristor at LR state due to (c) geometry-induced horizontal focusing and (d) optimal energy transfer; (e) optical transmission of the plasmonic memristor at HR and LR state; (f) extinction ration (ER) and insertion loss of the plasmonic memristor.

Table 1. Summary of the performances of some reported plasmonic memristors.

\begin{tabular}{ccccc}
\hline Refs & $\begin{array}{c}\text { Device Length } \\
(\boldsymbol{\mu} \mathbf{m})\end{array}$ & Loss $(\mathbf{d B})$ & $\begin{array}{c}\text { extinction Ratio } \\
(\mathbf{d B})\end{array}$ & $\begin{array}{c}\text { Extinction Efficiency } \\
(\mathbf{d B} / \boldsymbol{\mu m})\end{array}$ \\
\hline$[10]$ & 3 & 4.2 & 0.09 & 0.03 \\
{$[14]$} & $5(10)$ & $18(23)$ & $6(12)$ & 1.2 \\
{$[25]$} & 2.2 & 25 & 9.2 & 4.2 \\
{$[32]^{*} \#$} & 5 & 0.2 & 10 & 2 \\
{$[33]$} & 5000 & $/$ & 10 & 0.002 \\
{$[34]$} & 1000 & $/$ & 16 & 0.016 \\
This & 0.61 & 1 & 2.8 & 4.6 \\
work & & & & \\
\hline
\end{tabular}

\# Employ the epsilon-near-zero media between metal-like (plasmonic dispersion) and dielectric-like response ${ }^{*}$ numerical simulation results.
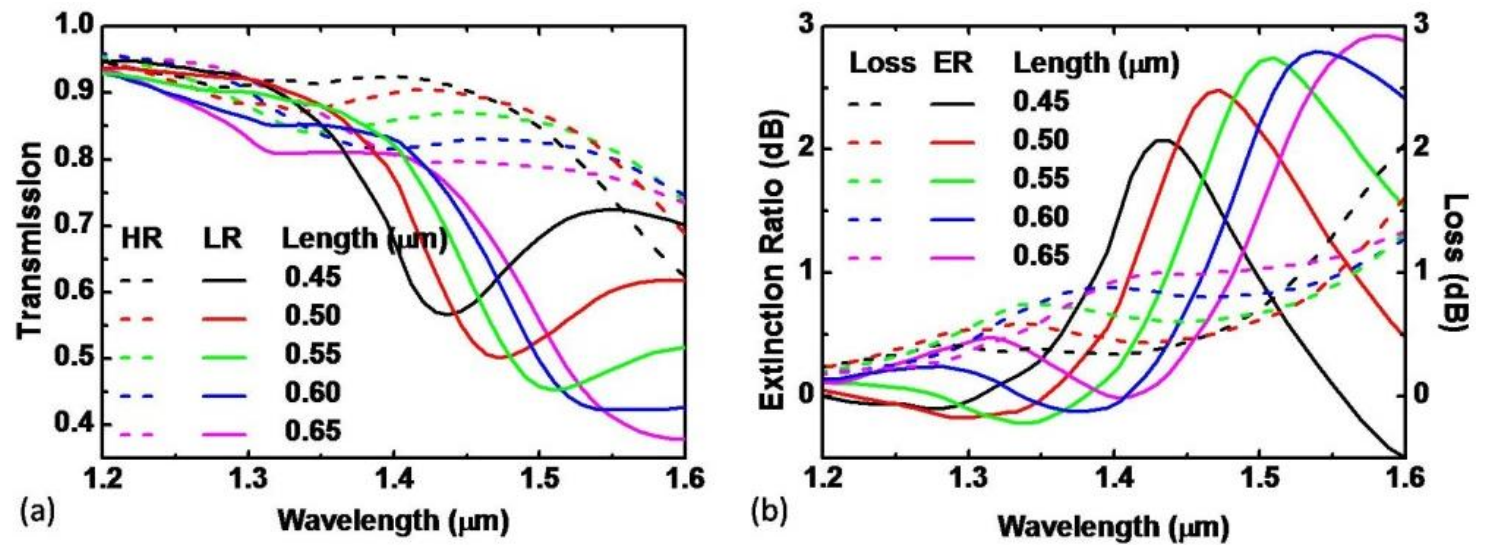

Figure 4. (a) Taper length dependence of the optical transmission of the plasmonic memristor at HR and LR states; (b) taper length dependence of the extinction ration (ER) and insertion loss of the plasmonic memristor. 


\subsection{Taper Length Dependence of the Properties of the Plasmonic Memristor}

Initially, we estimate the optimal taper length $(=791 \mathrm{~nm})$ by Equation (4), and simulate the optical response of this structure at both the LR and HR states. It founds a maximum extinction ratio at a relatively longer wavelength out of the telecommunication window. However, as the taper length is gradually reduced, a high ER at the C-band can be achieved, and, finally, we can obtain the best ER when the taper length is $610 \mathrm{~nm}$, as proposed above. To further study the influences of the taper length on our plasmonic memristor around the C-band, in this section, we discuss the transmission spectra of the plasmonic memristor (in both the HR and LR states) with different metallic taper lengths, from $0.65 \mu \mathrm{m}$ to $0.45 \mu \mathrm{m}$.

Figure 4a shows that the transmission $\mathrm{T}$ of the plasmonic memristor (at the HR state) is generally at a high level (from 0.95 to 0.6 , depending on the taper length) at wavelengths from $1.2 \mu \mathrm{m}$ to $1.6 \mu \mathrm{m}$. Accordingly, the insertion loss of the plasmonic memristor is typically $1 \sim 2 \mathrm{~dB}$ at the C-band (Figure $4 \mathrm{~b}$ ). Such a low loss in our design, compared with other reported structures, is due to the compact size, which reduces the intrinsic propagation loss of the plasmonic taper as discussed above; while, at the LR state, the transmission of the plasmonic memristor clearly decreases, indicating the remarkable extinction due to the formation of the filament (Figure 4a). When the taper length changes from $0.65 \mu \mathrm{m}$ to $0.45 \mu \mathrm{m}$, the maximum extinction ratio shifts from $1.43 \mu \mathrm{m}$ to $1.58 \mu \mathrm{m}$. This is because of the positive correlation between the operation wavelength and the optimal taper length, which can be quantitatively observed from Equation (4). However, along with the shorting of the taper length, the extinction ratio decreases, as shown in Figure $4 \mathrm{~b}$. This is ascribed to the subsiding of the geometry-induced horizontal focusing for the short taper. Actually, according to the adiabatic theory based on the WKB approximation [31], for the long taper with a small $d w / d x$, its adiabatic parameter $\delta$, defined by Equation (2), is accordingly small, and, subsequently, the geometric focusing of the light would be close to the ideal adiabatic focusing, while, for the shorter taper (large $d w / d x$ ), the $\delta$ increases, and thus the light concentration becomes non-adiabatic, i.e., not all the light input into the taper could be concentrated to its apex-some light energy leaks out of the taper and does not contribute to the plasmon-filament interaction [31].

\subsection{Filament Dependence of the Properties of the Plasmonic Memristor}

Other than the circular column filament with a radius of $10 \mathrm{~nm}$, considered in the aforementioned simulations, the filament in the cycled operations to the memristor has a different size and shape, and forms randomly at different sites of the insulator layer between the two electrodes [24,26]. The influences of these factors should be investigated. The recent work, based on kinetic Monte Carlo simulation, shows that the extinction behavior of the filament should be insensitive to its shape, but mainly governed by its size [32], so without the loss of generality, we fix the shape of the filament as the circular column, as conducted above, and check the extinction produced by the filament with a different radius, to evaluate the filament size-dependent properties of the plasmonic memristor. On the other hand, to understand the effects of the randomness of the filament site, which is a regular phenomenon existing in the memristor [24], we also study the variation in extinction due to the change in the position of the filament.

Figure 5a shows that for the taper with a length of $0.61 \mu \mathrm{m}$, as the filament radius decreases from $10 \mathrm{~nm}$ to $6 \mathrm{~nm}$, the extinction peak red-shifts remarkably and moves out of the C-band. As a result, the extinction ratio at $1.55 \mu \mathrm{m}$ decreases from $2.8 \mathrm{~dB}$ to $0.02 \mathrm{~dB}$ (accordingly the extinction efficiency changes from $4.6 \mathrm{~dB} / \mu \mathrm{m}$ to $0.033 \mathrm{~dB} / \mu \mathrm{m}$ ). Similarly, for the taper with a length of $0.45 \mu \mathrm{m}$, as the filament radius decreases from $10 \mathrm{~nm}$ to $6 \mathrm{~nm}$, the extinction peak red-shifts from $1.43 \mu \mathrm{m}$ to $1.53 \mu \mathrm{m}$, and the maximum extinction ratio changes from $2.08 \mathrm{~dB}$ to $0.65 \mathrm{~dB}$ (corresponding to the change in the extinction efficiency from $4.62 \mathrm{~dB} / \mu \mathrm{m}$ to $1.44 \mathrm{~dB} / \mu \mathrm{m}$ ). Therefore, it can be concluded that the extinction induced by the formation of the filament is positively correlated to its size. Meanwhile, it is noteworthy that the smaller the filament, the longer the peak wavelength of the extinction. The filament size dependence of the extinction behavior is very similar to 
the spectra features of the electrochemically programmable plasmonic antennas observed very recently [32]. Such a similarity indicates that the absorption and scattering of the filament to the plasmon, as proposed in previous research about the extinction mechanism of the plasmonic memristor $[10,14]$, may be highly affected by the transition of the localized resonant plasmonic mode (e.g., in the electrochemically programmable plasmonic antennas, the formation of the filament results in the transition from a bonding dipole plasmon to a charge transfer plasmon) [32]. Therefore, checking the possible influences of the resonance transition of the hybrid plasmon in the MIS structure might be beneficial for us to further improve the device's performance.

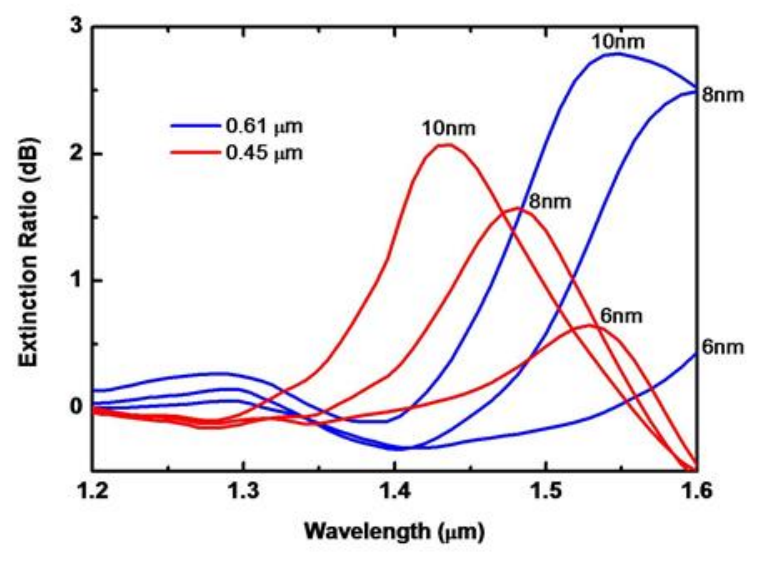

(a)

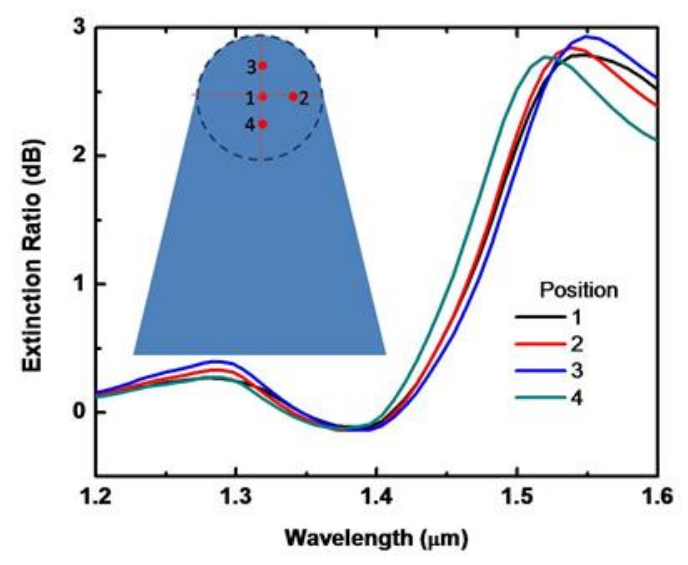

(b)

Figure 5. (a) Filament size dependence of the extinction ration (ER) of the plasmonic memristor; (b) filament position dependence of the optical transmission of the plasmonic memristor at HR and LR state.

Figure $5 \mathrm{~b}$ shows the extinction behavior that is dependent on the position of the filament. It was found that, for the filament with a fixed size, the closer its position to the apex, the higher the extinction ratio obtained. This is a quite natural result, since in our design, the electromagnetic energy of the plasmon is truly concentrated to the apex, due to the combination of geometry-induced horizontal focusing and optimal energy transfer from the $\mathrm{Si}$ waveguide to the plasmonic taper, as discussed above. However, other than the size of the filament, the extinction behavior of the plasmonic memristor is much less sensitive to the variation in the filament position. Therefore, practically, to achieve repeatable memristive operation of the plasmon, it is more critical to stabilize the filament size in comparison with reducing the randomness of the filament site. Fortunately, thanks to the increasingly deep understanding of the mechanisms of the memristor $[24,38,39]$, as well as the uninterrupted development of the strategy to control the filament growth in the memristors over the last few years [40,41], it is believable that the plasmonic memristor could become more repeatable and stable.

\section{Conclusions}

In this paper, we propose a design of a plasmonic memristor working at a telecom wavelength of $1.55 \mu \mathrm{m}$, with a compact size $(0.61 \mu \mathrm{m})$, low insertion loss $(1 \mathrm{~dB})$, and high extinction efficiency $(4.6 \mathrm{~dB} / \mu \mathrm{m})$. It consists of a triangle $\mathrm{Ag}$ taper integrated on the $\mathrm{Si}$ waveguide with a $\mathrm{SiO}_{2}$ buffer layer. In this structure, the $\mathrm{Ag}$ taper serves as both the top electrode of the memristor and light concentrator, due to the combination of geometryinduced horizontal focusing and optimal energy transfer to the taper. The rational doping to the $\mathrm{Si}$ waveguide in the area below the apex of the taper defines the bottom electrode and, accordingly, confines the zone in which the memristive behaviors (formation and rupture of the filament) occur in the area near the apex. As a result, the interactions between the plasmon and the filament in the memristor could be maximized. We point out that the key 
to improving the repeatable and stable plasmonic memristor is the effective control of the filament size. Our work might be valuable to improve the plasmonic memristor, as well as promote its application in on-chip optical memory, opto-electrical hybrid synapse, etc.

Author Contributions: Conceptualization, Y.T. and S.Z; simulations, Y.T.; writing-original draft preparation, Y.T.; writing — review and editing, Y.T., S.Z. and W.T. All authors have read and agreed to the published version of the manuscript.

Funding: This research was supported by NSFC (11947088, U1832143), Hunan Provincial Natural Science Fund (2019JJ50025) and the Scientific Research Fund of Hunan Provincial Education Department (No. 19B100).

Data Availability Statement: All data included in this study are available upon request by contact with the corresponding author.

Conflicts of Interest: The authors declare no conflict of interest.

\section{References}

1. Soref, R. The Past, Present, and Future of Silicon Photonics. IEEE J. Sel. Top. Quantum Electron. 2006, 12, 1678-1687. [CrossRef]

2. Melikyan, A.; Koehnle, K.; Lauermann, M.; Palmer, R.; Koeber, S.; Muehlbrandt, S.; Schindler, P.; Elder, D.; Wolf, S.; Heni, W.; et al. Plasmonic-organic hybrid (POH) modulators for OOK and BPSK signaling at 40 Gbit/s. Opt. Express. 2015, 23, 9938-9946. [CrossRef] [PubMed]

3. Melikyan, A.; Alloatti, L.; Muslija, A.; Hillerkuss, D.; Schindler, P.C.; Li, J.; Palmer, R.; Korn, D.; Muehlbrandt, S.; Van Thourhout, D.; et al. High-speed plasmonic phase modulators. Nat. Photon. 2014, 8, 229-233. [CrossRef]

4. Barnes, W.L.; Dereux, A.; Ebbesen, T.W. Surface plasmon subwavelength optics. Nature 2003, 424, 824-830. [CrossRef]

5. Tian, Y.; Jiang, L.; Deng, Y.; Deng, S.; Zhang, G.; Zhang, X. Bi-nanorod/Si-nanodot hybrid structure: Surface dewetting induced growth and its tunable surface plasmon resonance. Opt. Mater. Express 2015, 5, 2655-2666. [CrossRef]

6. Yang, J.; Perrin, M.; Lalanne, P. Analytical Formalism for the Interaction of Two-Level Quantum Systems with Metal Nanoresonators. Phys. Rev. X 2015, 5, 021008. [CrossRef]

7. Pile, D.F.; Ogawa, T.; Gramotnev, D.K.; Matsuzaki, Y.; Vernon, K.C.; Yamaguchi, K.; Okamoto, T.; Haraguchi, M.; Fukui, M. Two-dimensionally localized modes of a nanoscale gap plasmon waveguide. Appl. Phys. Lett. 2005, 87, 261114. [CrossRef]

8. Nikolajsen, T.; Leosson, K.; Bozhevolnyi, S.I. Surface plasmon polariton based modulators and switches operating at telecom wavelengths. Appl. Phys. Lett. 2004, 85, 5833-5835. [CrossRef]

9. Heeres, R.W.; Dorenbos, S.N.; Koene, B.; Solomon, G.S.; Kouwenhoven, L.P.; Zwiller, V. On-chip single plasmon detection. Nano Lett. 2009, 10, 661-664. [CrossRef] [PubMed]

10. Emboras, A.; Goykhman, I.; Desiatov, B.; Mazurski, N.; Stern, L.; Shappir, J.; Levy, U. Nanoscale Plasmonic Memristor with Optical Readout Functionality. Nano Lett. 2013, 13, 6151-6155. [CrossRef]

11. Strukov, D.B.; Snider, G.S.; Stewart, D.R.; Williams, S. The missing memristor found. Nature 2008, 453, 80-83. [CrossRef] [PubMed]

12. Mehonic, A.; Cueff, S.; Wojdak, M.; Hudziak, S.; Jambois, O.; Labbé, C.; Garrido, B.; Rizk, R.; Kenyon, A. Resistive switching in silicon suboxide films. J. Appl. Phys. 2012, 111, 074507. [CrossRef]

13. Tian, Y.; Guo, C.; Guo, S.; Yu, T.; Liu, Q. Bivariate-continuous-tunable interface memristor based on Bi2S3 nested nano-networks. Nano Res. 2014, 7, 953-962. [CrossRef]

14. Hoessbacher, C.; Fedoryshyn, Y.; Emboras, A.; Melikyan, A.; Kohl, M.; Hillerkuss, D.; Hafner, C.; Leuthold, J. The plasmonic memristor: A latching optical switch. Optica 2014, 1, 198. [CrossRef]

15. Di Martino, G.; Tappertzhofen, S.; Hofmann, S.; Baumberg, J. Nanoscale Plasmon-Enhanced Spectroscopy in Memristive Switches. Small 2016, 12, 1334-1341. [CrossRef]

16. Koch, U.; Hoessbacher, C.; Emboras, A.; Leuthold, J. Optical memristive switches. J. Electroceramics 2017, 39, 239-250. [CrossRef]

17. Borghetti, J.; Snider, G.S.; Kuekes, P.J.; Yang, J.J.; Stewart, D.R.; Williams, R.S. Memristive'switches enable 'stateful' logic operations via material implication. Nature 2010, 464, 873-876. [CrossRef]

18. Kvatinsky, S.; Kolodny, A.; Weiser, U.C.; Friedman, E.G. Memristor-based IMPLY logic design procedure. In Proceedings of the 2011 IEEE 29th International Conference on Computer Design (ICCD), Amherst, MA, USA, 9-12 October 2011; pp. 142-147.

19. Parra, J.; Olivares, I.; Brimont, A.; Sanchis, P. Non-volatile epsilon-near-zero readout memory. Opt. Lett. 2019, 44, 3932-3935. [CrossRef]

20. Singh, L.; Jain, S.; Kumar, M. Electrically writable silicon nanophotonic resistive memory with inherent stochasticity. Opt. Lett. 2019, 44, 4020-4023. [CrossRef]

21. Ríos, C.; Youngblood, N.; Cheng, Z.; Le Gallo, M.; Pernice, W.H.P.; Wright, C.D.; Sebastian, A.; Bhaskaran, H. In-memory computing on a photonic platform. Sci. Adv. 2019, 5, eaau5759. [CrossRef]

22. Xue, W.; Ci, W.; Xu, X.-H.; Liu, G. Optoelectronic memristor for neuromorphic computing. Chin. Phys. B 2020, $29,048401$. [CrossRef] 
23. Portner, K.; Schmuck, M.; Lehmann, P.; Weilenmann, C.; Haffner, C.; Ma, P.; Leuthold, J.; Luisier, M.; Emboras, A. Analog Nanoscale Electro-Optical Synapses for Neuromorphic Computing Applications. ACS Nano 2021, 15, 14776-14785. [CrossRef] [PubMed]

24. Waser, R.; Dittmann, R.; Staikov, G.; Szot, K. Redox-based resistive switching memories-nanoionics mechanisms, prospects, and challenges. Adv. Mater. 2009, 21, 2632-2663. [CrossRef]

25. Tian, Y.; Pan, L.; Guo, C.F.; Liu, Q. Atomic origin of the traps in memristive interface. Nano Res. 2016, 10, 1924-1931. [CrossRef]

26. Sawa, A. Resistive switching in transition metal oxides. Mater. Today 2008, 11, 28-36. [CrossRef]

27. Flammer, P.D.; Banks, J.M.; Furtak, T.E.; Durfee, C.G.; Hollingsworth, R.E.; Collins, R.T. Hybrid plas-mon/dielectric waveguide for integrated silicon-on-insulator optical elements. Opt. Express 2010, 18, 21013-21023. [CrossRef] [PubMed]

28. Luo, Y.; Chamanzar, M.; Adibi, A. Compact on-chip plasmonic light concentration based on a hybrid pho-tonic-plasmonic structure. Opt. Expres. 2013, 21, 1898-1910. [CrossRef]

29. Dong, P.; Liao, S.; Liang, H.; Shafiiha, R.; Feng, D.; Li, G.; Zheng, X.; Krishnamoorthy, A.V.; Asghari, M. Submilliwatt, ultrafast and broadband electro-optic silicon switches. Opt. Express 2010, 18, 25225-25231. [CrossRef] [PubMed]

30. Chen, Y.; Shu, Z.; Zhang, S.; Zeng, P.; Liang, H.; Zheng, M.; Duan, H. Sub-10 nm fabrication: Methods and applications. Int. J. Extreme Manuf. 2021, 3, 032002. [CrossRef]

31. Stockman, M.I. Nanofocusing of Optical Energy in Tapered Plasmonic Waveguides. Phys. Rev. Lett. 2004, 93, 137404. [CrossRef]

32. Dong, S.; Zhang, K.; Yu, Z.; Fan, J.A. Electrochemically Programmable Plasmonic Antennas. ACS Nano 2016, 10, 6716-6724. [CrossRef] [PubMed]

33. Ahn, Y.; Son, J.Y. The effect of size on the resistive switching characteristics of NiO nanodots. J. Phys. Chem. Solids 2016, 99, 134-137. [CrossRef]

34. Ma, Y.; Yeoh, P.P.; Shen, L.; Goodwill, J.M.; Bain, J.A.; Skowronski, M. Evolution of the conductive filament with cycling in TaOx-based resistive switching devices. J. Appl. Phys. 2020, 128, 194501. [CrossRef]

35. Emboras, A.; Niegemann, J.; Ma, P.; Haffner, C.; Pedersen, A.; Luisier, M.; Schimmel, T.; Leuthold, J. Atomic Scale Plasmonic Switch. Nano Lett. 2016, 16, 709-714. [CrossRef]

36. Matsuzaki, Y.; Okamoto, T.; Haraguchi, M.; Fukui, M.; Nakagaki, M. Characteristics of gap plasmon wave-guide with stub structures. Opt. Express. 2008, 16, 16314-16325. [CrossRef]

37. Singh, L.; Srivastav, S.; Kaushik, V.; Rajput, S.; Mishra, R.D.; Kumar, M. Light Assisted Electro-Metallization in Resistive Switch with Optical Accessibility. J. Light. Technol. 2021. [CrossRef]

38. Tian, Y.; Zhang, J.; Guo, C.F.; Zhang, B.; Liu, Q. Photoconductive probing of the trap distribution in switchable interfaces Nanoscale 2016, 8, 915-920. [CrossRef]

39. Pan, F.; Chen, C.; Wang, Z.-S.; Yang, Y.-C.; Yang, J.; Zeng, F. Nonvolatile resistive switching memo-ries-characteristics, mechanisms and challenges. Prog. Nat. Sci. Mater. Int. 2010, 20, 1-15. [CrossRef]

40. Wu, W.; Shan, B.; Feng, K.; Nan, H. Resistive switching behavior of Sb2S3 thin film prepared by chemical bath deposition. Mater. Sci. Semicond. Process. 2016, 44, 18-22. [CrossRef]

41. Nardi, F.; Ielmini, D.; Cagli, C.; Spiga, S.; Fanciulli, M.; Goux, L.; Wouters, D. Control of filament size and reduction of reset current below $10 \mu \mathrm{A}$ in $\mathrm{NiO}$ resistance switching memories. Solid State Electron. 2011, 58, 42-47. [CrossRef] 